\title{
Tuberculosis en pacientes ancianos. Formas de presentación
}

\author{
F. L. LADO LADO, V. TUÑEZ BASTIDA**, A. L. GOLPE GÓMEZ*, A. CABARCOS \\ ORTIZ DE BARRÓN, M. L. PÉREZ DEL MOLINO*** \\ Servicios de Medicina Interna, *Neumología y ***Microbiología. Complejo Hospitalario \\ Universitario de Santiago. Departamento de Medicina. **Unidad de Prevención y \\ Control de Tuberculosis del Área Sanitaria de Santiago de Compostela
}

TUBERCULOSIS IN EDERLY PATIENTS. FORMS OF PRESENTATION

\section{RESUMEN}

Objetivo: Analizar la distribución de las formas de presentación de tuberculosis (FPT) en pacientes de edad avanzada.

Material y métodos: Se revisaron los casos de tuberculosis registrados en la Unidad de Prevención y Control de la Tuberculosis del Área Sanitaria de Santiago de Compostela en un período de seis años. Clasificamos las FPT en: formas pulmonares (P), definida por localización exclusivamente pulmonar; Formas extrapulmonares (FE), caracterizada por una localización extrapulmonar; Formas mixtas (FM), localización pulmonar y extrapulmonar; Formas diseminadas (FD), dos o más localizaciones extrapulmonares; y TB miliares, definida por patrón radiológico miliar y/o enfermedad diseminada en necropsia.

Resultados: Se registraron un total de 278 tuberculosis, $156(56,2 \%)$ eran varones y $122(43,8 \%)$ mujeres. La edad media fue de 75,3 años (rango, 65-95). La distribución de FPT resultó ser: 155 (55,8\%) pulmonares; $66(23,7 \%)$ FE de las cuales $27(41,0 \%)$ eran de localización ganglionar, $12(18,2 \%)$ osteoarticular, $8(12,1 \%)$ intestinal, $6(9,1 \%)$ peritoneal, $5(7,6 \%)$ meningea y $8(12,0 \%)$ otras localizaciones; FM 47 casos $(16,9 \%)$; TB miliares 7 casos $(2,5 \%)$ y FD 3 casos $(1,1 \%)$. No se observó ningún caso de pacientes infectados por el virus de la inmunodeficiencia humana.

Conclusiones: En estos pacientes, se comprueba una elevada prevalencia de tuberculosis extrapulmonar. Ello nos sugiere, un cambio en la presentación clásica de la enfermedad y una extrema sensibilidad en el diagnóstico localizatorio de la enfermedad.

PALABRAS CLAVE: Tuberculosis. Extrapulmonar. Anciano.

\section{ABSTRACT}

Objetive: To analyse the distribution of the forms of presentation of tuberculosis $(T P F)$ in elderly patients.

Material and methods: The medical records of patients diagnosed with tuberculosis attending the Tuberculosis Prevention and Control Unit of the Santiago Health District were reviewed over of six years period. The classification of TPF was: pulmonary forms $(P)$, disease confined to the lung; extrapulmonary forms $(E F)$, disease outside the lung; mixed forms $(M F)$, the presence of both pulmonary and extrapul monary tuberculosis; disseminated forms $(D F)$, the pressence of two or more extrapulmonary locations; and miliary $T B$, which was definied by a diffuse pulmonary radiographic pattern or diagnosis was undertaken by necropsy.

Results: A total of 278 tuberculosis infected patients were observed, $156(56.2 \%)$ were men and $122(43.8 \%)$ women, their mean age was 75.3 years (range 65-95). The distribution of TPF was: 155 (55.8\%) $P$ forms; $66(23.7 \%) \mathrm{EF}$, of which $27(41.0 \%)$ were ganglionary location, $12(18.2 \%)$ bone and joint, $8(12.0 \%)$ intestinal, $6(9.1 \%)$ peritoneal, 5 (7.6\%) meningeal, and other locations 8 (12.1\%); MF 47 cases (16.9\%); miliary TB 7 cases $(2.5 \%)$ and. DF 3 cases $(1.1 \%)$. None case was obser ved of HIV infected patient.

Conclusions: Our findings confirm high incidence of extrapulmo nary $T B$ in elderly patients. Our experience shows a modification to the classical presentation of the disease, and thus the need for sensitivity in locating the disease.

KEY WORDS: Tuberculosis. Extrapulmonary. Elderly.

Lado Lado FL, Tuñez Bastida V, Golpe Gómez AL, Cabarcos Ortíz de Barrón A, Pérez del Molino ML. Tuberculosis en pacientes ancianos. Formas de Presentación. An Med Interna (Madrid) 2002; 19: 111-114.

\section{INTRODUCCIÓN}

En el momento actual, la tuberculosis sigue siendo un problema sociosanitario relevante incluso en países desarrollados. Diversos factores, entre ellos la irrupción de la infección producida por el virus de la inmunodeficiencia humana (VIH) (1-7), los cambios demográficos y los integrados por ciertos grupos marginales (como los indigentes) y los hábitos tóxicos (la drogadicción y el alcoholismo), han contribuído a un des- censo del control de la tuberculosis (8-12). Asimismo, asistimos a un incremento de la tuberculosis en pacientes de edad avanzada debido, no sólo, al aumento de la población geríatrica, sino también, a otros elementos como son la reactivación de focos endógenos previos o la progresividad de infecciones adquiridas en instituciones cerradas (13-18).

En la población inmunocompetente, la forma de presentación habitual de la tuberculosis es la pulmonar. Así, en el año 1986 de 22.764 casos declarados en EE.UU. el $82,5 \%$ eran

Trabajo aceptado: 27 de noviembre de 2001

Correspondencia: F.L. Lado Lado. Servicio de Medicina Interna. Hospital Clínico Universitario. C/ A Choupana s/n. 15706 Santiago de Compostela. A Coruña. 
pulmonares mientras que el $17,5 \%$ restante tenían presentación extrapulmonar, representada principalmente por las localizaciones ganglionar, pleural y génitourinaria (19). Sin embargo, la tuberculosis en los pacientes de edad avanzada adquiere una serie de características especiales con respecto a otros grupos de edad. Así, existe un alto riesgo de mostrar formas crípticas o diseminadas, o un menor indicio radiológico de cavitación en su presentación pulmonar. En consecuencia, ello puede conducir a una elevada morbimortalidad en este grupo derivado, quizás, de un retraso en el diagnóstico y de la frecuente asociación con otras enfermedades intercurrentes en este grupo poblacional $(16,17,20-24)$.

El objetivo del presente estudio es conocer la distribución, en nuestro medio, de las distintas formas de presentación de la enfermedad tuberculosa en pacientes de edad avanzada, a partir de los casos registrados en una unidad de prevención y control de tuberculosis durante un período de seis años.

\section{MATERIAL Y MÉTODOS}

Se trata de un análisis retrospectivo en el que se revisan los casos de enfermedad tuberculosa registrados en la Unidad de Prevención y Control de la Tuberculosis del Área Sanitaria de Santiago de Compostela que abarca una población, urbana y rural, estimada en aproximadamente 435.000 habitantes. El período de estudio comprende entre Febrero de 1995 y Febrero de 2001.

En el presente estudio se incluyeron todos aquellos casos diagnosticados de tuberculosis con edad igual o superior a lo 65 años mediante los criterios que detallamos a continuación: A) Presencia de bacilos ácido alcohol resistentes (BAAR) mediante la tinción de Ziehl-Nielsen o de auramina-rodamina y/o cultivo positivo para Mycobacterium tuberculosis en medio de Löwestein-Jensen en una o más muestras biológicas. B) Evidencia de granulomas con necrosis caseosa en los estudio histológicos procedentes de las distintas muestras obtenidas por punción aspiración con aguja fina (PAAF) o por biopsia.

Se recogieron como datos referentes a factores predisponentes los relativos a los antecedentes conocidos de estado serológico frente al VIH, alcoholismo, enfermedades del tejido conectivo, y antecedentes de entidades y/o procesos que, en su curso, tuvieran implícitos datos de inmunosupresión tales como: insuficiencia renal crónica, neoplasias, enfermedad pulmonar obstructiva crónica, diabetes mellitus y tratamiento prolongado con corticoides.

Se clasificaron las distintas formas de presentación de la tuberculosis en las siguientes categorías: Formas pulmonares, definida por localización exclusivamente pulmonar; Formas extrapulmonares, caracterizado por una única localización extrapulmonar; Formas mixtas, localización pulmonar y extrapulmonar; Formas diseminadas, dos o más localizaciones extrapulmonares; y TB miliares, definidas por patrón miliar en la radiografía de tórax y/o enfermedad diseminada en necropsia.

En el análisis estadístico se introdujeron los datos en hoja de cálculo Excel (ver. 97). Se emplearon las medias, porcentajes y comparación de proporciones.

\section{RESULTADOS}

En el período objeto de análisis, un total de 1661 casos de tuberculosis fueron declarados de los cuales un total de 278
$(16,7 \%)$ se correspondieron con pacientes de edad igual o superior a los 65 años de edad. De estos 278 casos, 156 $(56,2 \%)$ eran varones y $122(43,8 \%)$ mujeres. La edad media fue de 75,3 años (rango, 65-95) siendo mayoritario el grupo comprendido entre los 65 y 75 años (Tabla I). Un total de 40 casos $(14,4 \%)$ presentaba algunos factores predisponentes, ya fuera de forma aislada o combinada, los más comunes fueron: alcoholismo en 15 casos $(37,5 \%)$, Diabetes mellitus en 8 $(20,0 \%)$, insuficiencia renal crónica en $7(17,5 \%)$, silicosis en $4(10,0 \%)$, y otras en 6 casos $(15,0 \%)$ (Tabla I). No se registró ningún caso de infección por el virus de la inmunodeficiencia humana.

\section{TABLA I}

\begin{tabular}{lr}
\multicolumn{2}{c}{ TABLA I } \\
\hline \multicolumn{1}{c}{$\mathrm{N}$ D $(\%)$} \\
\hline Edad media (rango) & $75,3(65-95)$ \\
Grupos de edad (años) & \\
$65-75$ & $166(59,7)$ \\
$76-85$ & $95(34,2)$ \\
$* 86$ & $17(6,1)$ \\
& \\
Sexo & \\
Varón & $156(56,2)$ \\
M ujer & $122(43,8)$ \\
& \\
Factores predisponentes Y EPIDEM IO LÓ GICO S & \\
Alcoholismo & $15(37,5)$ \\
Diabetes mellitus & $8(20,0)$ \\
O tros & $17(42,5)$ \\
\hline
\end{tabular}

№ = Número de casos.

La distribución de las distintas formas de presentación de la tuberculosis resultó ser: Pulmonares con una suma de 155 $(55,8 \%)$ casos; Formas extrapulmonares con un total 66 casos $(23,7 \%)$ que se distribuyeron como sigue: 27 (41,0\%) Ganglionar, $12(18,2 \%)$ Osteoarticular, 8 (12,1\%) Intestinal, 6 $(9,1 \%)$ Peritoneal, $5(7,6 \%)$ Meningea, y otras localizaciones $8(12,0 \%)$ que se repartieron del siguiente modo: Pleural en 2 casos, Génitourinaria en 2, Pericárdica en 2, Cutánea en 1 y Área ORL en 1; Formas mixtas con un total de 47 casos (16,9\%), siendo predominante (29 casos) la localización pulmonar y ganglionar intratorácica $(61,7 \%)$; TB miliares en 7 casos $(2,5 \%)$. En tres de ellos, el diagnóstico se efectuó por necropsia; y Formas diseminadas en un total de 3 casos $(1,1 \%)$ (Figs. 1,2).

\section{DISCUSIÓN}

En nuestro medio, la tuberculosis se caracteriza por tener una elevada incidencia. Así, entre los años 1989 y 1994 osciló entre 65,8 y 95,5 casos por 100.000 habitantes $(25,26)$. En el año 1995, se registró una incidencia de 67,4 casos por 100.000 habitantes (27) y, entre los años 1996 y 1998 fluctuó entre 61,9 


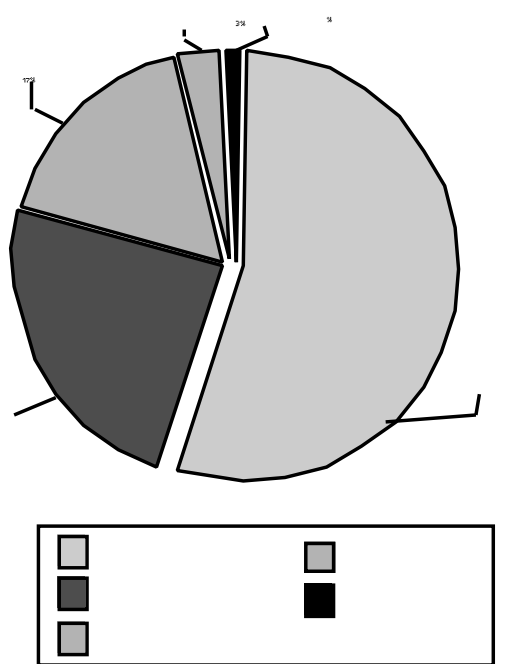

Fig. 1. FPT declaradas (FPT: formas de presentación de la tuberculosis).

y 78,3 casos por 100.000 habitantes (28). Por tal motivo, a nivel sociosanitario existe un alto grado de preocupación en lo que supone un amplio conocimiento y estrecho control sobre la repercusión de la enfermedad tuberculosa (29).

Los factores predisponentes de relevancia, al contrario de otros estudios en que encuentran con mayor frecuencia a las enfermedades neoplásicas o la hipertensión arterial y las enfermedades cardiovasculares como procesos asociados $(17,21)$, resultaron ser el alcoholismo y la Diabetes mellitus, no recogiéndose, como era de esperar por el grupo de edad objeto de análisis, ningún caso de infección por el VIH, la cual adquiere especial relevancia en los grupos comprendidos entre los 20 y 49 años $(7,30)$.

En el presente estudio, la tuberculosis como enfermedad pulmonar, ya sea aislada o asociada a otras localizaciones, fue la más habitual, como era de esperar. En cuanto a los hallazgos radiológicos en esta forma de presentación, cabe destacar que en consonancia con lo descrito por otros autores que reflejan una baja frecuencia de cavitación en este grupo poblacional con respecto a otros $(17,24)$, en el presente se evidenció en el $16,3 \%$ de los casos. Con respecto a la localización extrapulmonar que, clásicamente venía a representar una estimación inferior al $20 \%$ en la población inmunocompetente (19), la encontramos superior a dicho porcentaje, hecho ya decrito y conocido en nuestra experiencia $(31,32)$, lo cual nos hace suponer que, muy probablemente, se esté modificando el comportamiento de la tuberculosis, habida cuenta que en la presente serie no hubo constancia de infección por el VIH.

Dentro de las formas extrapulmonares, pudimos verificar que la ganglionar fue la más usual presentándose en un $41,0 \%$ y, a distancia, hallamos un 18,2\% de localización osteoarticular. Estos resultados contrastan con los obtenidos por otros autores $(19,33)$ que dan preferencia a la localización ganglionar en grupos de edad más jóvenes mientras que la localización osteoarticular se corresponde a edades avanzadas. En nuestra serie, encontramos en orden de prevalencia, la localización intestinal que no suele ser tan frecuente en la experiencia de otros autores (33), seguida de las localizaciones peritoneal y meningea.

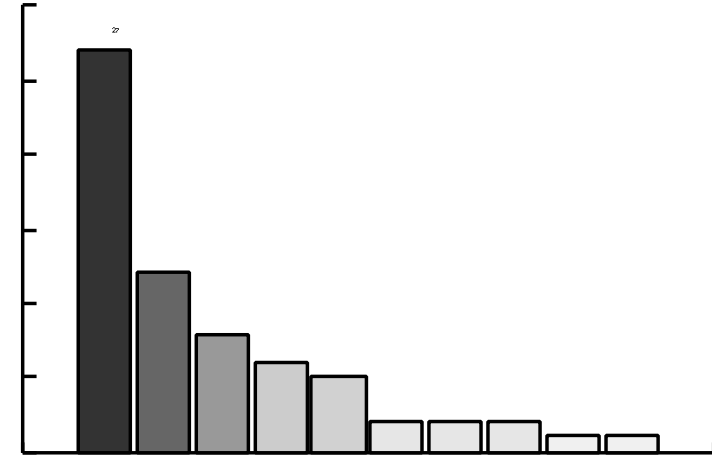

Fig. 2. Formas extrapulmonares declaradas.

En lo referente a la peritonitis tuberculosa, ésta se evidenció en seis casos y aunque se considera común en todos los grupos de edad, es más frecuente en etapas avanzadas de la vida $(19,34,35)$. Por el contrario, la meninigitis tuberculosa, presente en cinco casos, suele ser más frecuente en grupos de edad más jóvenes que incluyen la población pediátrica $(33,36)$. En lo que respecta a la localización pleural, ésta puede acontecer a cualquier edad pero es más habitual $(60 \%)$ en grupos inferiores a los 30 años (37), y así lo constatamos en la presente muestra en la que sólamente registramos dos casos. En cuanto a las formas restantes, a diferencia de otros autores (19), que describen un incremento de la localización génitourinaria según aumenta la edad, nosotros encontramos una baja incidencia.

Con respecto a las formas mixtas, que incluyen participación pulmonar y extrapulmonar, resultaron las más usuales después de las formas de localización única extrapulmonar con una representación del 16,9\%. En este grupo cabe destacar que, si bien no suele ser un hecho frecuente la afectación ganglionar intratorácica $(17,22)$, la hemos observado en más de la mitad de los casos $(61,7 \%)$ de estas formas de presentación de la tuberculosis. También es interesante recalcar que, en los pacientes infectados por el VIH, en nuestra experiencia dicha asociación representa, después de las formas limitadas al pulmón, la contemplada con mayor frecuencia (7).

La tuberculosis miliar, se encuentra en tercer lugar, después de las formas mixtas, con un total de 7 casos $(2,5 \%)$, tres de los cuales fueron diagnosticados en necropsia. En este sentido, se ha apreciado una mayor incidencia de la tuberculosis miliar en edades avanzadas y pacientes inmunodeprimidos con presentación clínica atípica, que implican una extrema dificultad diagnóstica si no se sospecha la enfermedad y, de hecho, un porcentaje nada despreciable de casos son diagnosticados en necropsia $(33,38-41)$.

Las formas diseminadas aparecieron en tres casos alcanzando la forma de presentación menos prevalente. En estas formas, al igual que otros autores y tal como hemos realizado en anteriores ocasiones $(32,42)$, se incluyen aquellos casos de más de una localización extrapulmonar con el fin de separarlos de aquellos considerados como tuberculosis miliar. 
Como resumen de los expuesto, comentamos que las formas pulmonares han sido las más frecuentemente registradas. En las formas extrapulmonares la localización constatada con mayor reiteración ha sido la ganglionar, seguida de la osteoarticular. Las formas mixtas, se presentaron asociadas a la localización ganglionar intratorácia, en la mayoría de los casos.
Para finalizar, destacamos que la presentación extrapulmonar de la tuberculosis en este grupo de edad en nuestro medio, supera las expectativas esperables. Ello nos indica por una parte, un cambio significativo en la presentación clásica de la enfermedad y, por otra una extrema sensibilidad en cuanto al diagnóstico localizatorio de la enfermedad.

\section{Bibliografía}

1. Raviglione MC, Snider DE, Kochi A. Global epidemiology of tuberculosis. Morbidity and mortality of a worldwide epidemic. JAMA 1995; 273: 220-6.

2. Shafer RW, Edlin BR. Tuberculosis in patients infected with immunodeficiency virus: perspective on the past decade. Clin Infect Dis 1996; 22: 683-704

3. Murray JF. Tuberculosis and HIV infection: global perspectives. Respirology 1997; 3: 209-13

4. Martínez Vazquez JM, Cabarcos Ortíz de Barrón A, Barrio Gómez E. Tuberculosis e infección por VIH: epidemiología (primera de tres partes). An Med Interna (Madrid) 1997: 14: 253-6.

5. Martínez Vazquez JM, Cabarcos Ortíz de Barrón A, Barrio Gómez E. Tuberculosis e infección por VIH: patogenia (segunda de tres partes). An Med Interna (Madrid) 1997; 14: 310-6.

6. Martínez Vazquez JM, Cabarcos Ortíz de Barrón A, Barrio Gómez E. Tuberculosis e infección por VIH: de la clínica a la prevención (tercera de tres partes). An Med Interna (Madrid) 1997: 14: 363-8.

7. Lado Lado FL, Barrio Gómez E, Carballo Arceo E, Cabarcos Ortíz de Barrón A. Tuberculosis e infección por el virus de la inmunodeficencia humana: manifestaciones clínicas y rendimiento de procedimientos diagnósticos según las distintas formas de localización de la enfermedad. An Med Interna (Madrid) 2000; 17: 27-34.

8. Brudney K, Dobkin J. Resurgent tuberculosis in New York City. Human immunodeficiency virus, homelessness, and the decline of tuberculosis control programs. Am Rev Respir Dis 1991; 144: 745-9.

9. Cantwell MF, Snider DE, Cauthen GM, Onorato IM. Epidemiology of tuberculosis in the United States, 1985 through 1992. JAMA 1994; 272 . 535-9.

10. Zolopa AR, Hahn JA, Gorter R, Miranda J, Wlodarczyk D, Peterson J, et al. HIV and tuberculosis infection in San Francisco's homeless adults. Prevalence and risk factors in a representative sample. JAMA 1994; 272: 455-61.

11. Del Rey Calero. Incremento actual de la tuberculosis. An Med Interna (Madrid) 1995; 12: 209-11.

12. Pablos-Méndez A, Knirsch ChA, Barr RG, Lerner BH, Frieden ThR Nonadherence in tuberculosis treatment: predictors and consequences in New York city. Am J Med 1997; 102: 164-70.

13. Stead WW. Pathogenesis of first episode of chronic pulmonary tuberculosis in man: recrudescence of residuals of prymary infection of exogenous reinfection. Ann Intern Med 1968; 68: 731-45.

14. Stead WW, Lofgren JP. Does the risk of tuberculosis increase in old age? J Infect Dis 1983; 147: 951-5.

15. Stead WW, Lofgren JP, Warren E, Thomas C. Tuberculosis as an endemic and nosocomial infection among the elderly in nursing homes. $\mathrm{N}$ Engl J Med 1985; 312: 1483-7.

16. Stead WW, Dutt AK. Tuberculosis in the elderly. Semin Respir Infect 1989; 4: 189-97.

17. Korzeniewska-Kosela M, Krysl J, Müller N, Black W, Allen E, FitzGerald JM. Tuberculosis in young adults and the elderly. A prospective comparison study. Chest 1994; 106: 28-32.

18. Duffield JS, Adams WH, Anderson M, Leitch AG. Increasing incidence of tuberculosis in young and the elderly in Scotland. Thorax 1996; 51: 140-2.

19. Rieder HL, Snider DE, Cauthen GM. Extrapulmonary tuberculosis in the United States. Am Rev Respir Dis 1990; 141: 347-51.

20. Fulton JD, McCallioin J. Tuberculosis-diagnostic difficulty in the elderly. J Clin Exp Gerontol 1987; 9: 303-311.

21. Umeki S. Comparison of younger and elderly patients with pulmonary tuberculosis. Respiration 1989; 55: 75-83.

22. Davies PD. Tuberculosis in the elderly. J Antimicrob Chemother 1994; 34 (Suppl A): 93-100.

23. Rieder HL, Kelly GD, Bloch AB, Cauthen GM, Snider DE. Tuberculosis diagnosed at death in the United States. Chest 1991; 100: 678-811.

24. Zamarrón C, Salgueiro M, Álvarez JM, Otero Y, Rodríguez Súarez JR. Características clínicas de la tuberculosis pulmonar en el anciano. An Med Interna (Madrid) 1997; 14: 167-9.

25. Salgueiro Rodríguez M, Zamarron Sanz C, Álvarez-Calderon Prat P, et al. Estudio epidemiológico de la tuberculosis en el área sanitaria de Santiago de Compostela durante los años 1989, 1990 y 1991. An Med Interna (Madrid) 1993; 10: 427-32.

26. Salgueiro M, Zamarron C, Otero Y, et al. Estudio epidemiológico de la tuberculosis en el área sanitaria de Santiago de Compostela durante los años 1992, 1993 y 1994. An Med Interna (Madrid) 1996; 13: 111-4.

27. Tuñez Bastida MV, Golpe Gómez AL, Paniagua López J. La enfermedad tuberculosa en el área sanitaria de Santiago durante el año 1995. Arch Bronconeumol 1997; 33 (Suppl 1): S 32.

28. Salgueiro Rodríguez M, Zamarron C, González Barcala J, et al. Estudio epidemiológico de la tuberculosis en el área sanitaria de Santiago de Compostela durante los años 1995,1996, 1997 y 1998. An Med Interna (Madrid) 2001; 18: 20-3

29. Anibarro García L, Vázquez-Gallardo R, Toubes Navarro ME, Penas Truque A, Lema Mougán R, Túñez Bastida V, et al. Epidemiología de la tuberculosis en Galicia. An Med Interna (Madrid) 1999; 16: 290-6.

30. Shafer RW, Kim DS, Weiss JP, Quale JM. Extrapulmonary tuberculosis in patients with immunodeficiency virus infection. Medicine 1991; 70: 384-97.

31. Cowie RL, Sharpe JW. Extra-pulmonary tuberculosis: a high frecuency in the absence of HIV infection. Int J Tuberc Lung Dis 1997; 2: 159-62.

32. Lado Lado FL, Túñez Bastida V, Golpe Gómez A, Ferreiro Regueiro MJ, Cabarcos Ortiz de Barrón A. Tuberculosis extrapulmonar. Formas de presentación en nuestro medio. An Med Interna (Madrid) 2000; 17: 637-41.

33. Alvarez S, McCabe WR. Extrapulmonary tuberculosis revisited: a review of experience at Boston and others hospitals. Medicine 1984; 63: $25-55$.

34. Karney WW, O'Donoghue JM, Ostrow JH, Holmes KK, Beaty HN. The spectrum of tuberculous peritonitis. Chest 1977; 72: 310-5

35. González Anglada MI, Barbado Hernández FJ, Cano Ruiz A, et al Tuberculosis peritoneal. Características de una norara localización tuberculosa. Rev Clin Esp 1992; 190: 393-7.

36. Ribera E, Ocaña I, Martínez Vázquez JM, Gallofre M, Fernández de Sevilla T, Reñe R. Características clínicas y dificultades diagnósticas de 35 casos de meningitis tuberculosa. Neurología (Esp.) 1987; 2: 11-8.

37. Haro M, Ruiz-Manzano J, Gallego M, Abad J, Manterola JM, Morera J. Tuberculosis pleural: análisis de 105 casos. Enferm Infecc Microbiol Clin 1996; 14: 285-9.

38. Conthe P, Gaspar G, Redondo C, et al. El problema de la tuberculosis miliar: estudio de 20 casos no diagnosticados hasta la autopsia. Med Clin (Barc) 1982; 79: 268-72.

39. Kim JH, Langston AA, Gallis HA. Miliary tuberculosis: epidemiology, clinical manifestations, diagnosis, and outcome. Rev Infect Dis 1990; 12: 583-90.

40. De Miguel, Encinar L, Villanueva R, García Rego J, Freire R, Diz Lois F. Tuberculosis miliar en adultos. Estudio en 67 pacientes. An Med Interna (Madrid) 1990; 7: 5-12.

41. Veiga González M, Riestra Martínez M, Fresno Forcelledo M, González González M, Ablanedo Ablanedo P, Herrero Zapatero A. Tuberculosis miliar. Estudio autópsico de 29 casos. An Med Interna (Madrid) 1995; 12: 17-20.

42. Hill AR, Premkumar S, Brustein S, et al. Disseminated tuberculosis in the acquired immunodeficiency syndrome era. Am Rev Respir Dis 1991; 144: 1164-70. 